\title{
Experimental plant communities develop phylogenetically overdispersed abundance distributions during assembly
}

\author{
Eric Allan, ${ }^{1,2,9}$ Tania Jenkins, ${ }^{1,3}$ Alexander J. F. Fergus, ${ }^{4}$ Christiane Roscher,${ }^{5,6}$ Markus Fischer, ${ }^{2}$ \\ Jana Petermann, ${ }^{4,7}$ Wolfgang W. Weisser,${ }^{8}$ and Bernhard Schmid ${ }^{4}$ \\ ${ }^{1}$ Institute of Ecology, Friedrich Schiller University of Jena, Dornburger Straße 159, 07749 Jena, Germany \\ ${ }^{2}$ Institute of Plant Sciences, University of Bern, Altenbergrain 21, 3013 Bern, Switzerland \\ ${ }^{3}$ Department of Ecology and Evolution, University of Lausanne Sorge, Le Biophore CH-1015 Lausanne, Switzerland \\ ${ }^{4}$ Institute of Evolutionary Biology and Environmental Studies, University of Zürich, Winterthurerstr. 190, 8057 Zürich, Switzerland \\ ${ }^{5}$ Max Planck Institute for Biogeochemistry, Hans-Knöll Straße 10, 07745 Jena, Germany \\ ${ }^{6}$ UFZ, Helmholtz Centre for Environmental Research, Department of Community Ecology, Theodor-Lieser-Straße 4, \\ 06120 Halle, Germany \\ ${ }^{7}$ Institute of Biology, Freie Universität Berlin, Königin-Luise-Straße 1-3, 14195 Berlin, Germany \\ ${ }^{8}$ Department of Ecology and Ecosystem Management, Technische Universität München, 85354 Freising, Germany
}

Abstract. The importance of competition between similar species in driving community assembly is much debated. Recently, phylogenetic patterns in species composition have been investigated to help resolve this question: phylogenetic clustering is taken to imply environmental filtering, and phylogenetic overdispersion to indicate limiting similarity between species. We used experimental plant communities with random species compositions and initially even abundance distributions to examine the development of phylogenetic pattern in species abundance distributions. Where composition was held constant by weeding, abundance distributions became overdispersed through time, but only in communities that contained distantly related clades, some with several species (i.e., a mix of closely and distantly related species). Phylogenetic pattern in composition therefore constrained the development of overdispersed abundance distributions, and this might indicate limiting similarity between close relatives and facilitation/complementarity between distant relatives. Comparing the phylogenetic patterns in these communities with those expected from the monoculture abundances of the constituent species revealed that interspecific competition caused the phylogenetic patterns. Opening experimental communities to colonization by all species in the species pool led to convergence in phylogenetic diversity. At convergence, communities were composed of several distantly related but species-rich clades and had overdispersed abundance distributions. This suggests that limiting similarity processes determine which species dominate a community but not which species occur in a community. Crucially, as our study was carried out in experimental communities, we could rule out local evolutionary or dispersal explanations for the patterns and identify ecological processes as the driving force, underlining the advantages of studying these processes in experimental communities. Our results show that phylogenetic relations between species provide a good guide to understanding community structure and add a new perspective to the evidence that niche complementarity is critical in driving community assembly.

Key words: biodiversity; community assembly; convergence; Jena Experiment; limiting similarity; phylogenetic dispersion.

\section{INTRODUCTION}

A major question in ecology is what drives community assembly. There is still much debate about the relative importance of limiting similarity or environmental filtering, with analyses based on functional traits giving different results in natural communities (Stubbs and Wilson 2004, Thompson et al. 2010). The evolutionary history of species has long been used to

Manuscript received 21 December 2011; revised 19 July 2012; accepted 14 September 2012. Corresponding Editor: B. D. Inouye.

${ }^{9}$ E-mail: eric.allan@ips.unibe.ch understand community assembly (e.g., Darwin 1859) but has recently received increased attention (Webb et al. 2002, Cadotte et al. 2008, Cavender-Bares et al. 2009, Vamosi et al. 2009). Using phylogenetic relations between species to understand their interactions has the advantage that phylogeny may integrate information on hard-to-measure traits, such as the co-evolved enemies shared between species, that would not be included in functional trait measures (Kraft and Ackerly 2010). Phylogenetic patterns in community composition can indicate the ecological processes underlying community assembly: phylogenetic overdispersion, where the species present are distantly related, is expected to arise from limiting similarity processes, which prevent 
closely related species from coexisting (Pacala and Tilman 1994). Phylogenetically clustered compositions, where the species present are closely related, are often interpreted as being caused by environmental filtering on phylogenetically conserved species traits (Vamosi et al. 2009). However, competition could also cause this pattern if competitive ability itself is phylogenetically conserved (Mayfield and Levine 2010). Quantifying the importance of competition for driving phylogenetic patterns is therefore important for understanding the mechanisms behind them.

The vast majority of studies on phylogenetic pattern have examined the presence/absence of species in a community (composition) but not their local abundances (Hardy 2008, Vamosi et al. 2009), meaning they have ignored later stages of community assembly. However, the species that dominate a community may not be a random sample, with respect to their functional traits, of those present (Cornwell and Ackerly 2010), and different processes can determine which species become abundant vs. those that establish at a site (Cingolani et al. 2007). Other studies have shown that different phylogenetic patterns may be found when incorporating data on species occurrence frequency (Kembel 2009, Kraft and Ackerly 2010) or abundance (Hardy and Senterre 2007). Phylogenetic overdispersion may increase during succession (Webb et al. 2006, Letcher 2009) and some studies have shown a greater importance of environmental filtering in early successional communities (Helmus et al. 2010). Therefore species might shift their relative abundances during community assembly, so that the dominant species in a community become less closely related over time, i.e., abundance distributions become increasingly overdispersed.

Several studies have shown that the type of phylogenetic pattern found in a community depends on the phylogenetic scale: overdispersion should be more common in communities with close relatives present (Cavender-Bares et al. 2006, Swenson et al. 2006), where negative species interactions, i.e., competition, are expected to dominate (Burns and Strauss 2011). On the other hand, complementary (Cadotte et al. 2008, Gubsch et al. 2011) or facilitative (Valiente-Banuet and Verdu 2007) interactions may be more common between distantly related species. The presence of several distantly related clades in a community, each containing a number of species (a mix of closely and distantly related species), might therefore promote overdispersed abundance distributions. To test the influence of phylogenetic scale, the development of phylogenetic pattern in abundance distributions could be compared between communities containing species compositions fixed at different phylogenetic diversities, with the prediction that species abundances would only become overdispersed in communities containing distantly related clades each with several species (see Fig. 1). This idea of an interaction between phylogenetic pattern in community composition and the phylogenetic pattern in abundance distribution that develops has not yet been tested.

Allowing artificial plant communities to reassemble has been shown to lead to convergence in functional, species (Pfisterer et al. 2004, Fukami et al. 2005, Petermann et al. 2010) and phylogenetic diversity (Cadotte and Strauss 2011). A recent study showed that the species that established in reassembling communities tended to be either closely or distantly related to the residents (Cadotte and Strauss 2011). Allowing reassembly in composition to occur alongside assembly of abundances allows us to test whether communities converge on phylogenetic compositions that result in phylogenetically overdispersed abundance distributions. If communities converge at overdispersed or clustered compositions there might be no phylogenetic pattern in abundance distributions (Fig. 1a, b) because phylogenetically based environmental filtering/limiting similarity has already occurred, so the strength of species interactions are not correlated with the phylogenetic distance between them. Alternatively, random phylogenetic compositions might result in overdispersed abundance distributions if closely related species can co-occur within a community but cannot both reach high abundance (Fig. 1c).

Biodiversity experiments provide an ideal opportunity to test these ideas because they contain replicate plots with a range of species numbers and compositions, the latter determined by a random draw from a species pool. It is therefore possible to study the development of phylogenetic pattern as these communities reassemble, while excluding local evolutionary or dispersal explanations (Cavender-Bares et al. 2009), something previous observational studies could not do. Here, we examine the development of phylogenetic patterns in the Jena Experiment, a grassland biodiversity experiment in Germany that manipulated species richness and functional group composition (Roscher et al. 2004). We calculated changes in phylogenetic pattern in abundance distributions for experimental communities over seven years, using a measure called abundance phylogenetic dispersion (APD; Hardy 2008), which quantifies whether the abundant species in a community are more or less closely related than the average. APD is a relative measure and is independent of phylogenetic pattern in composition, i.e., even communities composed of closely related species can in principle develop overdispersed abundance distributions.

We investigate changes in phylogenetic pattern in abundance distribution during two processes of community assembly or reassembly: first, using weeded communities with fixed species composition where phylogenetic pattern was only affected by changes in the relative abundances of species. Here we test for the interaction between phylogenetic pattern in species' presence/absence and the phylogenetic pattern in abundance distribution that develops. We also calculate the importance of interspecific competition for the develop- 
Species pool

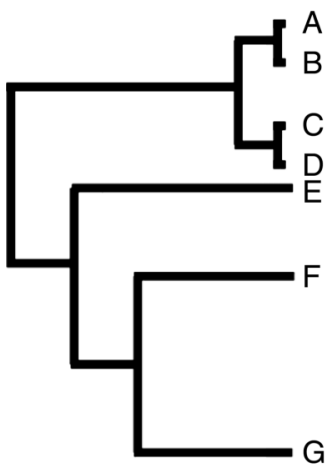

Local communities

a

Only close relatives present

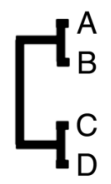

b

Only distant relatives present

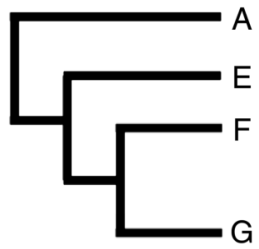

C

A mix of close and distant relatives present

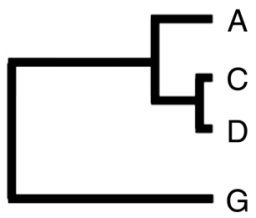

Only negative interactions

A $B$ B $C$ D

A

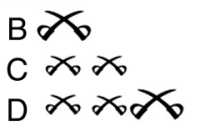

Neutral or positive interactions (or interaction strength not correlated with phylogeny)

A $E F G$

A

$E \Omega$

$F$ sas

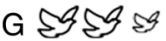

A mix of negative and positive interactions

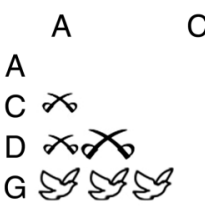

Variable phylogenetic pattern in abundance distribution

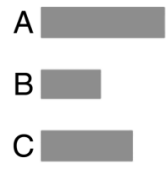

D

No phylogenetic pattern in abundance distribution

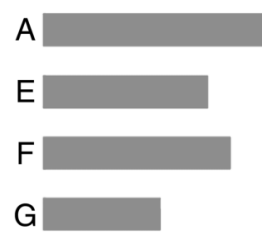

Overdispersed abundance distribution

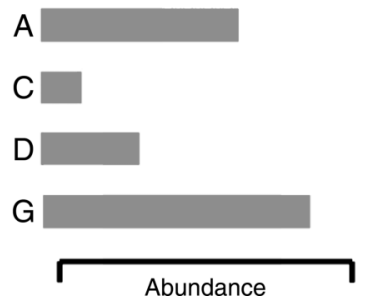

FIG. 1. The hypothesized effect of phylogenetic pattern in species' presence/absence in a community on the emergence of phylogenetic pattern in abundance distribution. The phylogeny of the species pool is shown on the left. Species have been assembled from the pool into three types of communities: (a) a community with clustered composition, i.e., only close relatives present; (b) a community with overdispersed composition, i.e., only distant relatives present; and (c) a community with random phylogenetic composition, containing distantly related clades but with some clades having multiple species, i.e., a mix of close and distant relatives. Hypothesized species interactions are shown on a matrix for each community: crossed swords represent negative interactions and doves represent positive or neutral interactions, larger symbols show stronger interactions. The consequences of these interactions for species abundances are shown on the right in bar plots. Where the composition is clustered or overdispersed (communities a or b) then the abundance distribution is less likely to be determined by phylogenetic relations between species because environmental filtering/limiting similarity has already determined community composition, and therefore the phylogenetic distances between species present do not predict the strength of their interactions. If the composition is phylogenetically random (community c) then there is more scope for species abundances to shift to reduce negative interactions between close relatives, and overdispersion in abundance distributions can develop.

ment of phylogenetic pattern in abundance distribution by comparing the pattern expected for a community based on the abundance of its species in monoculture (i.e., without interspecific competition) with that observed in the presence of interspecific competition. Using monocultures to infer the importance of complementary species interactions is the basis of the additive-partitioning method (Loreau and Hector 2001) and here we use an analogous approach to look at the importance of species interactions in driving phylogenetic pattern. Second, we investigate changes in phylogenetic pattern during the reassembly of communities following colonization by species from a common species pool; here species composition changes alongside abundances.
Using the unique opportunity of a wide range of different plant communities composed of a common species pool and situated at a homogeneous field site, we examine the following hypotheses:

1) Over time communities become overdispersed in abundance and this is driven by interspecific competition.

2) In fixed-composition communities, the phylogenetic pattern in composition will affect the development of overdispersion in abundance distributions (see also Fig. 1).

3) Functional, and at the same time phylogenetic, groups with strong complementary interactions with other species (here: legumes) promote overdispersion. 


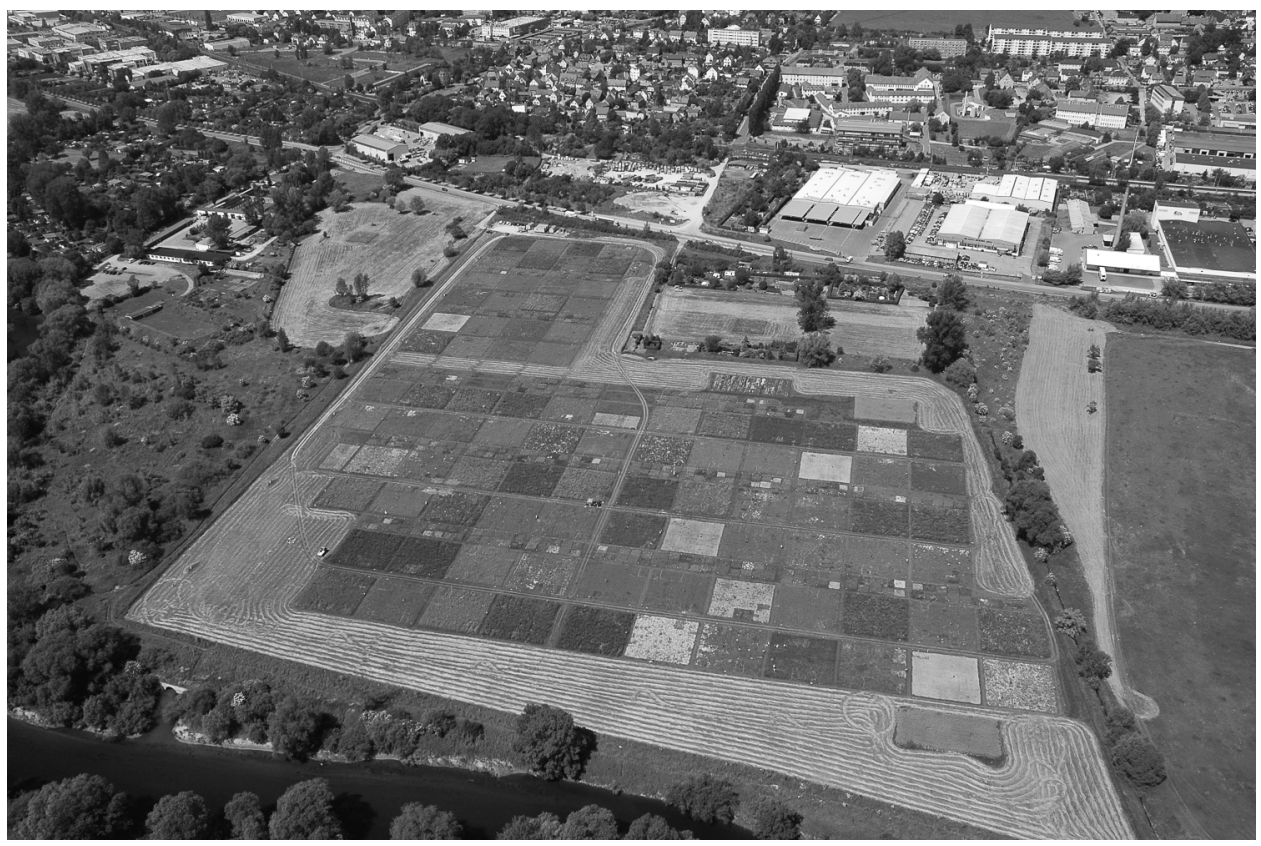

Plate 1. Aerial view of the Jena Experiment in June 2006. The $20 \times 20 \mathrm{~m}$ plots of the fixed composition experiments are clearly visible. The subplots of the reassembly experiment, opened to colonization in April 2005, are visible as differently shaded squares within some of the large plots. Photo credit: Alexandra Weigelt.

4) Allowing species composition to reassemble along with changes in abundance will result in convergence in phylogenetic diversity and overdispersion in abundance distributions.

\section{Methods}

\section{The Jena Experiment}

The Jena Experiment (see Plate 1) has 78 large plots $(20 \times 20 \mathrm{~m})$ with $1,2,4,8$, or 16 plant species, selected from a pool of 60 plant species. Plants belong to four functional groups (FG): grasses, legumes, small herbs, and tall herbs (Roscher et al. 2004). Number and presence of FGs was varied systematically, e.g., plots with four or more species could have 1, 2, 3, or 4 FGs. In addition, monocultures of each species were grown on $3.5 \times 3.5 \mathrm{~m}$ plots. Plots were sown with $1000 \mathrm{seeds} / \mathrm{m}^{2}$, equally divided among the species present and adjusted to species germination rates (Roscher et al. 2004). In this fixed-composition experiment species composition was held constant (except for extinctions) through biannual weeding in early April and July, when all species not sown into the plot initially were removed. Individual species-cover data were collected twice yearly, in spring and summer, on a subplot of $3 \times 3 \mathrm{~m}$. Biomass was harvested at the same time and sorted to species. For more details on sampling see Weigelt et al. (2010). For the main analyses here we use cover data from 20022009. Using cover means we estimate species abundances over a larger area than biomass sampling would have allowed, providing a better estimate of the abundance of less common species. For our calculations, we used only plots containing 4-16 species (46 plots) as it is not meaningful to calculate a phylogenetic pattern in the abundance of only one or two species.

We also investigated phylogenetic pattern in a reassembly experiment carried out in all 78 plots; monocultures and two-species plots were included because species numbers rapidly increased during reassembly (Roscher et al. 2009a). In this experiment, seeds of all 60 species were sown, in April 2005, at equal proportions and at a total density of 1000 viable seeds/ $\mathrm{m}^{2}$, into the existing vegetation in subplots of $2.00 \times 2.25$ $\mathrm{m}$. Species not belonging to the species pool continued to be weeded out from July 2005. Cover estimations were made on the whole area of these subplots at the same time as in the large plots, using an identical protocol. Pre-2005 data came from two subplots of the same size weeded as the large plots (Roscher et al. 2009b).

\section{Phylogeny reconstruction}

We searched GenBank in March 2009 and again in June 2012 for four gene sequences commonly used in building angiosperm phylogenies (Benson et al. 1999). We used closely related congeners for 2 of the 60 species for which there were no available sequence data (see Fig. 2). Each species used for the phylogeny reconstruction had sequence data for at least one gene and we had data for $\mathrm{rbcl}$ ( $90 \%$ of species), matk ( $97 \%$ of species), $5.8 \mathrm{~s}$ 


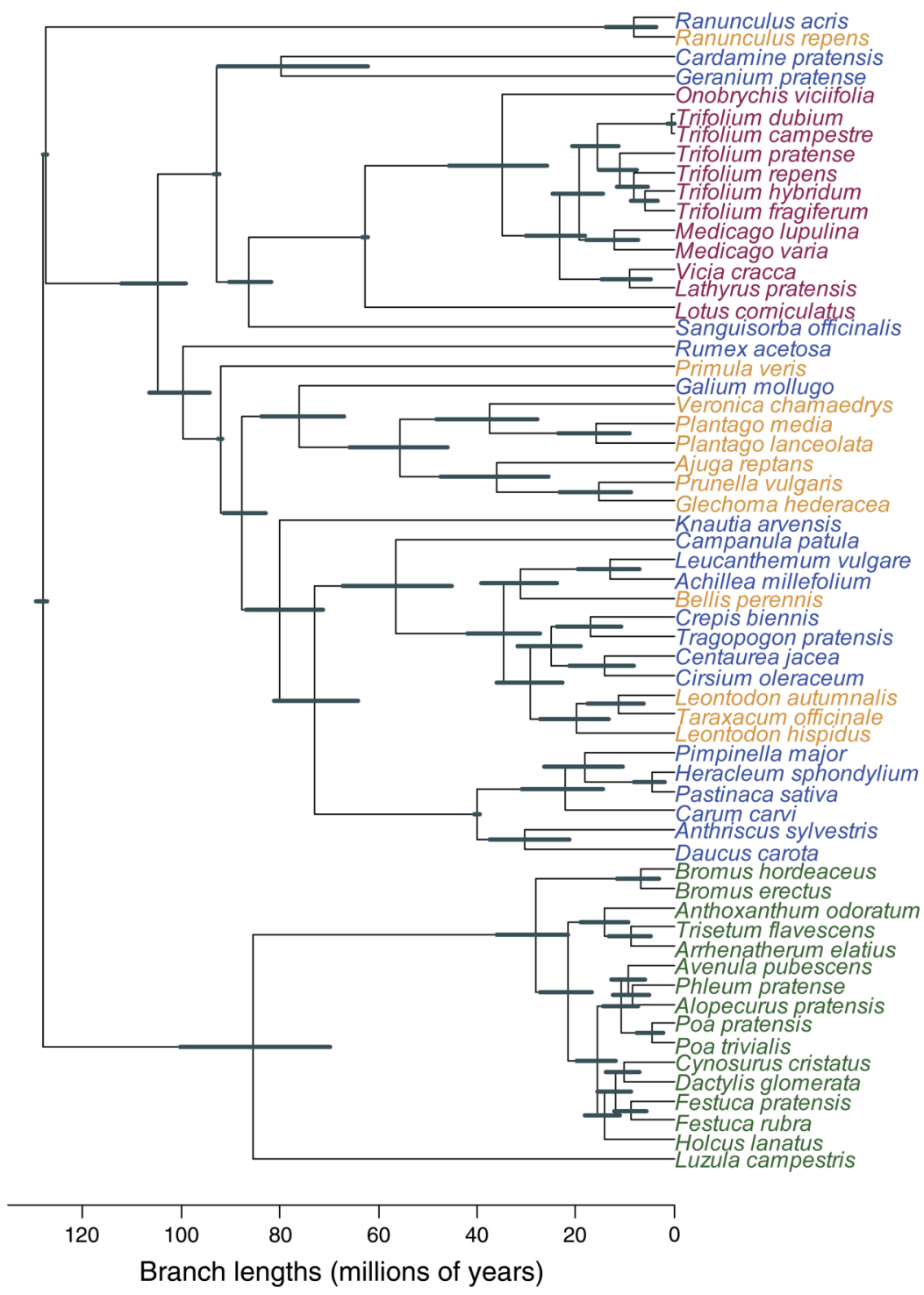

FIG. 2. Maximum clade-credibility phylogeny of the 60 species in the Jena Experiment. Different functional groups are differently colored: graminoids in green, legumes in yellow, small herbs in red, and tall herbs in blue. The $95 \%$ confidence intervals for node ages are shown. Congeners were used for Onobrychis viciifolia $(O$. montana) and for Pimpinella major ( $P$. saxifraga). Node support was high: $66 \%$ of nodes gave a posterior probability of 1 , and a further $23 \%$ a posterior probability $>0.97$. Only seven nodes were less well supported, four in the Poaceae, plus the placement of Bellis perennis (0.64), Rumex acetosa (0.68), and the node between Cardamine pratensis and Geranium pretense (0.82).

( $75 \%$ of species), and its 2 ( $92 \%$ of species), resulting in a total sequence length of 3581 base pairs. Sequences were individually aligned for each gene, separately per plant family, in MUSCLE (Edgar 2004). We used jModeltest (Posada and Crandall 1998) to test for models of DNA substitution for each gene separately, resulting in the selection of GTR $+\Gamma$.

We performed dated Bayesian reconstructions and estimates of divergence times using BEAST version 1.7.2 (Drummond and Rambaut 2007), with Amborella trichopoda and Magnolia grandiflora as outgroups. To obtain a dated molecular phylogeny we used six fossils: for the root of the tree (all angiosperms) and for the following groupings according to the Angiosperm Phylogeny Group tree (APG III 2009): Eudicots, Asterids, Rosids, Apiales and Fabaceae (Appendix A: Table A1). Parameters were estimated using two independent Markov chain Monte Carlo (MCMC) chains, each run for 26 million generations, and sampled every 1000 generations. Analyses were partitioned across the two mitochondrial genes, but the same site model was used for the two nuclear genes. We used a relaxed molecular clock model allowing branch lengths to vary according to an uncorrelated lognormal distribution and 

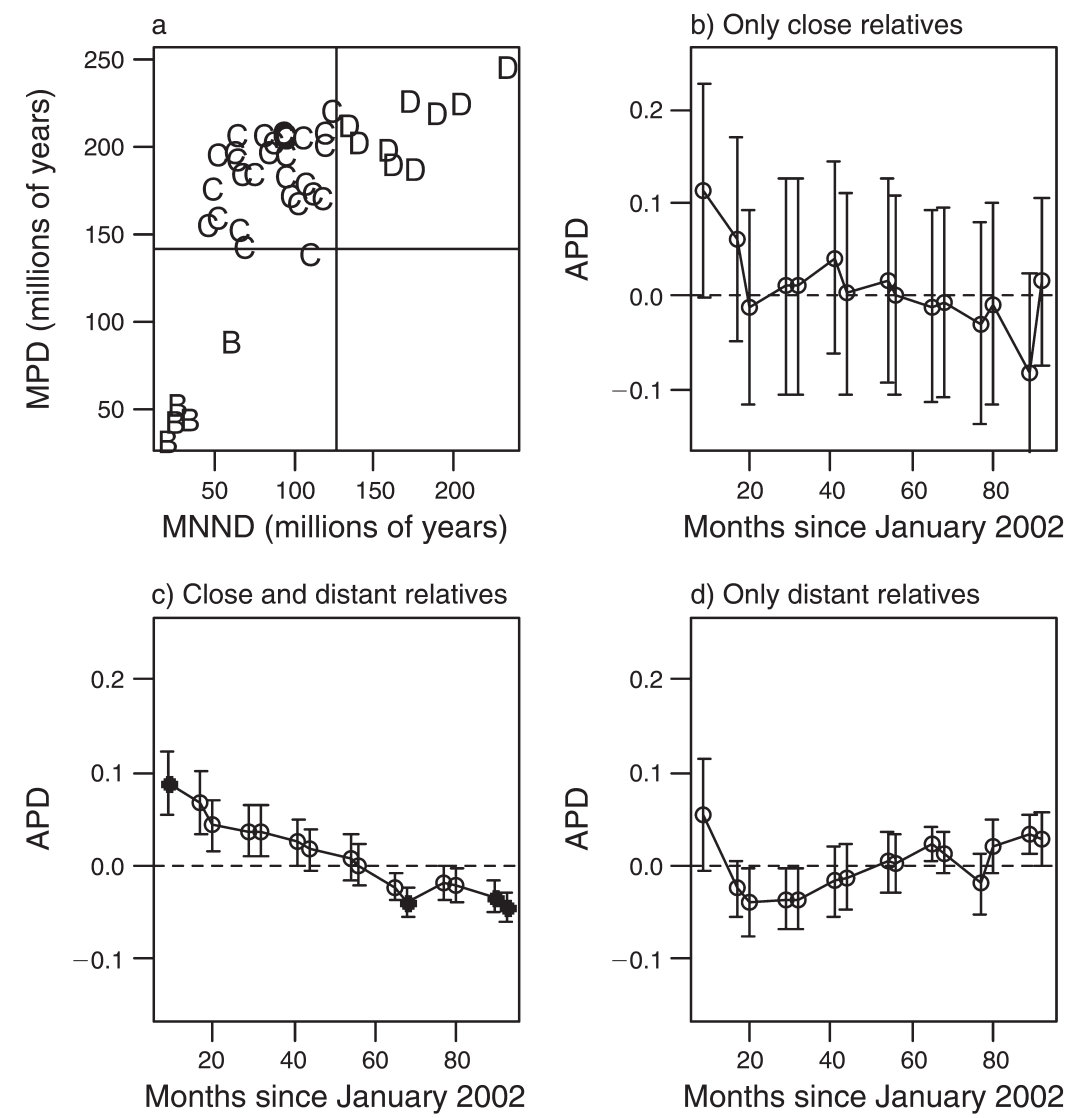

FIG. 3. Change in abundance phylogenetic dispersion (APD) over time in the fixed-composition experiment. Negative values of APD indicate overdispersed abundance distributions, and positive values indicate clustered abundance distributions. (a) Relationship between sown MPD (mean pairwise distance) and MNND (mean nearest neighbor distance) across plots. Plots were classified into three groups based on sown phylogenetic diversity: (b) those with MPD $<141$ million years (Myr) and MNND < $126 \mathrm{Myr}$; (c) MPD > $141 \mathrm{Myr}$ and MNND < $126 \mathrm{Myr}$; and (d) MPD > $141 \mathrm{Myr}$ and MNND > 126 Myr. In panel (a) these groups are indicated by uppercase letters (B-D); cut-off points (solid lines) are the midpoints in the range of MPD or MNND values and were used for illustration only; MPD and MNND were analyzed as continuous variables. In panels (b)-(d) open circles show plots with random phylogenetic structure (APD not significantly different from 0), and solid circles show those with significant phylogenetic structure (APD significantly different from 0). Data are means \pm SE; significance is at the $5 \%$ level.

a Yule speciation tree prior. Convergence and burn-in was assessed using Tracer version 1.5 (Drummond and Rambaut 2007), by inspection of parameter values and their associated likelihoods and by estimation of effective sample size (ESS) (ESS > 200 indicates convergence; Drummond et al. 2006). Tree files were combined using TreeAnnotator version 1.4.8 (Drummond and Rambaut 2007), with the first $10 \%$ of trees discarded as burn-in, in order to produce a posterior distribution of trees. Outgroups were removed from the trees and $10 \%$ of those in the posterior distribution (4680) were used in subsequent analyses.

\section{Sown phylogenetic diversity}

In order to test the interaction between phylogenetic pattern in composition and the development of phylogenetic pattern in abundance distributions (Fig. 1), we calculated two measures of phylogenetic diversity based on the sown species composition of the plots in 2002 .
Mean pairwise distance (MPD) measures the mean phylogenetic distance between all pairs of species and is affected by the number of deeper splits in the phylogeny. Mean nearest neighbor distance (MNND) measures the mean distance between each species and its closest relative and measures dispersion at the tips of the phylogeny (Webb et al. 2002). Both were calculated using picante (Kembel et al. 2010) in R 2.10 ( $\mathrm{R}$ Development Core Team 2010) for each plot using 4680 trees. In order to account for uncertainties in phylogenetic reconstruction, median as well as lower $(25 \%)$ and upper $(75 \%)$ quartile values were calculated.

Sown MPD and MNND were significantly positively correlated across communities; but several had high MPD and low MNND (Fig. 3a), indicating the presence of distantly related clades each with several species, i.e., a mix of close and distant relatives. MPD was unrelated to species richness, while MNND was lower in speciesrich communities (Appendix C: Fig. C1). The correla- 
tions between sown phylogenetic and species diversity provide the motivation for fitting all factors in our models (see Statistical analysis, below): we always fitted sown MPD and MNND as explanatory variables, meaning they did not change over time and were not affected by any species losses.

\section{Phylogenetic pattern based on species abundance distributions}

We calculated phylogenetic pattern in abundance distributions using APD (Hardy 2008), which quantifies the extent to which closely related species have similar abundance. For details on the calculation of this metric see Appendix B: Eqs. B.1 and B.2, or Hardy (2008). An APD $<0$ means overdispersed abundance distributions because it indicates that the most abundant species are more distantly related to each other than are the average pair of species. An APD $>0$ indicates clustered abundance distributions, meaning abundant species are closely related. As APD is a relative measure, even communities with low MPD can show overdispersed abundance.

For the fixed-composition and the reassembly experiments we calculated APD for all plots, years, and cover surveys, using 4680 trees, and median as well as lower $(25 \%)$ and upper $(75 \%)$ quartile values were obtained. The APD was calculated relative to MPD between all sown species in a plot. In order to test for significant phylogenetic pattern across plots we tested whether average APD significantly differed from 0; if average APD across plots did not significantly differ from 0 we considered them to have random phylogenetic structure in abundance.

\section{Effect of interspecific competition on phylogenetic pattern}

In order to test for the importance of interspecific competition in driving phylogenetic pattern, we used monoculture data to calculate a measure we call " $D_{\text {diff }}^{\mathrm{B}}$ "; for details on the calculation of this metric see Appendix B and Eq. B.3. The $D_{\text {diff }}^{\mathrm{B}}$ will be negative if the most abundant species in mixture are more phylogenetically distant from each other than are the most abundant species in monoculture, this would suggest that competition drives overdispersion in abundance. The $D_{\text {diff }}^{\mathrm{B}}$ will be positive if the most abundant species in mixture are more closely related than are the most abundant species in monoculture; this would indicate that interspecific competition results in phylogenetically clustered compositions.

\section{Statistical analysis}

To study effects of sown phylogenetic diversity, species richness and $\mathrm{FG}$ composition on change in phylogenetic pattern over time we used linear mixedeffects models fitted with the lme4 package in $\mathrm{R}$ (Bates and Sarkar 2007). The same model was used for data from the fixed-composition and the reassembly experiments. Models included a random effect for plot (46 plots in the fixed composition and 78 plots in the reassembly experiment) and a random effect for cover survey coded as a categorical factor (15 time points for the fixed composition and 6 time points for the reassembly experiment). Fixed effects were: time (continuous variable), sown species richness (log-transformed), sown MPD and sown MNND, FG composition (fitted as the presence/absence of each FG), and interactions between these terms (Appendix C: Tables $\mathrm{C} 1$ and $\mathrm{C} 2$ ). We simplified full models by removing nonsignificant terms and used likelihood-ratio tests to compare models with and without the term of interest (Crawley 2007).We tested for an effect of season by comparing a model with season and interactions between season, species richness, phylogenetic diversity, and FG composition (23 terms) with a model without any season terms (15 terms). In all cases the simpler model was preferred and seasonal effects are therefore not considered further. To test for a main effect of time we compared a model with a linear continuous term for time as the only fixed effect, with an intercept-only model. We analyzed the change in $D_{\text {diff }}^{\mathrm{B}}$ over time in mixed models with the same random effects as above and with fixed effects for time and interactions between time and sown species richness, sown MPD, and sown MNND.

To examine whether plots had random phylogenetic structure we fitted intercept-only models to test whether mean values across plots differed from 0 . We did this for each time period (cover survey or biomass harvest) for APD values in the reassembly and fixed-composition and for $D_{\text {diff }}^{\mathrm{B}}$ in the fixed-composition experiment.

We repeated all analyses using APD, MPD, and MNND values from $25 \%$ or $75 \%$ quartiles to correct for phylogenetic uncertainty. This led to the same qualitative results.

\section{RESUlts}

\section{Phylogeny reconstruction}

The phylogenetic reconstructions and the divergence time estimations converged in the same likelihood space and gave well-supported trees that agreed with the Angiosperm Phylogeny Group (APG) III classification (APG III 2009) (see Fig. 2 for the maximum clade credibility tree).

Phylogenetic pattern in the fixed-composition experiment

Species had initially been sown at equal proportions, so at sowing APD (abundance phylogenetic dispersion) $=0$. In the fixed-composition experiment, phylogenetic pattern in abundance distributions changed over time $\left(\chi^{2}=24.0 ; P<0.001\right)$ : communities had developed clustered abundance distributions by the first cover survey $(2002, \mathrm{APD}=0.09 \pm 0.03[$ mean $\pm \mathrm{SE}] ; P<$ 0.01 ) but after five years average APD was negative, i.e., abundant species were on average less closely related to each other than less abundant ones. 
Phylogenetic pattern in species composition-sown MPD (mean pairwise distance) and sown MNND (mean nearest neighbor distance) - affected the development of phylogenetic pattern in abundance distributions (Appendix $\mathrm{C}$ : Table $\mathrm{C} 1$ ). In order to visualize these effects, communities were divided into three groups: those sown with only close relatives (low MPD and MNND), those sown with distantly related but species-rich clades (a mix of close and distant relatives; low MNND but high MPD) and those sown without close relatives (high MPD and MNND) (Fig. 3a). In communities with only closely related species APD decreased over time $\left(\chi^{2}=8\right.$; $P<0.01)$ but there was large variability between plots and they ended up with, on average, random phylogenetic structure in abundance distributions (Fig. 3b). In communities containing distantly related but speciesrich clades, APD decreased over time $\left(\chi^{2}=33 ; P<\right.$ $0.001)$ and abundance distributions were overdispersed by the end of the time series (Fig. 3c). In communities with only distantly related species, APD did not change over time $\left(\chi^{2}=2.7 ; P=0.09\right)$ and phylogenetic pattern in abundance remained random (Fig. 3d).

Functional group composition affected the development of phylogenetic pattern in abundance distributions as communities with legumes became more rapidly overdispersed (Appendix C: Table C1). There were also significant interactions between presence of grasses, small herbs, tall herbs and time (Appendix C: Table C1). Plots with small herbs had lower APD values at the beginning of the experiment but higher APD at the end. Plots with grasses and plots with tall herbs were less overdispersed at the end of the experiment. The functional composition, as well as the phylogenetic diversity, of the community therefore affected the degree of overdispersion. The species richness of the community had no effect on the development of phylogenetic pattern in abundance distributions although species-rich plots were slightly less overdispersed (Appendix C: Table C1).

$D_{\text {diff }}^{\mathrm{B}}$ (which compares the observed phylogenetic pattern in abundance distribution for a community with that expected based on the monoculture abundances of the species) decreased over time, which is what would be predicted if competition drove the increase in overdispersion $\left(\chi^{2}=24 ; P<0.001\right.$; Appendix B: Fig. B1). This pattern was strongest in the 16-species plots (sown diversity $\times$ time interaction, $\chi^{2}=19 ; P<0.001$ ). At the beginning of the experiment $D_{\text {diff }}^{\mathrm{B}}$ was significantly greater than 0 , indicating that the abundant species in mixture were more closely related to each other than were the most abundant species in monoculture: i.e., interspecific competition resulted in phylogenetically clustered abundance distributions. By the end of the time series $D_{\text {diff }}^{\mathrm{B}}$ was significantly smaller than 0 , meaning abundant species were more distantly related in mixture than in monoculture. This indicates that interspecific competition drove an increase in phylogenetic overdispersion in abundance distributions.

\section{Phylogenetic pattern in the reassembly experiment}

In the reassembly experiment, communities became, on average, clustered in the first two surveys after colonization (spring 2005, APD $=0.06 ; P=0.01$; and autumn 2005, APD $=0.04 ; P=0.05)$ and overdispersed in abundance in spring $2006(\mathrm{APD}=-0.02 ; P=0.02)$. In subsequent surveys plots were not significantly overdispersed on average; however this was due to a single former legume monoculture that became entirely dominated by grasses and therefore highly clustered in abundance relative to all species that could colonize the community (see Fig. 4a). Excluding this plot, communities were also overdispersed in autumn 2007 (APD = $-0.02 ; P=0.01)$. Calculating APD ignoring phylogenetic pattern in the colonizing species led to similar patterns, but there was stronger evidence for overdispersion from spring 2006 onward and weaker evidence for clustering immediately after colonization (see Appendix D: Fig. D1).

In the course of the experiment, communities converged in phylogenetic diversity. By spring 2007 the range of $A P D$ values had contracted (Fig. 4a). MPD and MNND converged more rapidly: at convergence MPD was higher but MNND was lower than mean sown values (Fig. 4b and c). Therefore species composition converged so that plots ended up containing distantly related but species-rich clades.

The originally sown phylogenetic diversity of the communities affected the change in phylogenetic pattern immediately after colonization (Appendix C: Table C2). To explore this result, plots were classified as having sown (in 2002) MPD and MNND higher, lower, or in the range of $95 \%$ of values to which communities had converged by summer 2007 (Fig. 4b, c). MPD and MNND increased following colonization on plots with sown MPD or MNND lower than converged values and MPD and MNND decreased following colonization on plots with sown MPD and MNND higher than converged values. Classifying plots by sown MPD and MNND gave seven combinations but factor-level reduction (in a mixed model testing for differences in the slope of APD over time for these different categories) led to four categories, and change in phylogenetic pattern was then analyzed in these groups of communities separately. (1) Seven plots had been sown with only close relatives (sown MPD and MNND lower than converged values): here APD became strongly clustered after invasion and then decreased (time effect $\chi^{2}=8 ; P<0.01$ ) (Fig. 4d). (2) Nineteen plots had been sown with some more distantly related species (sown MPD lower; sown MNND higher or in the range of converged values) and these became less clustered after invasion than the first set of plots, before APD decreased (time effect $\chi^{2}=9 ; P<0.01$ ) (Fig. 4e). (3) Nineteen plots had been sown with distantly related but species-rich clades (MPD and MNND in the range of converged values) and here phylogenetic pattern was random after colonization and then decreased (time 

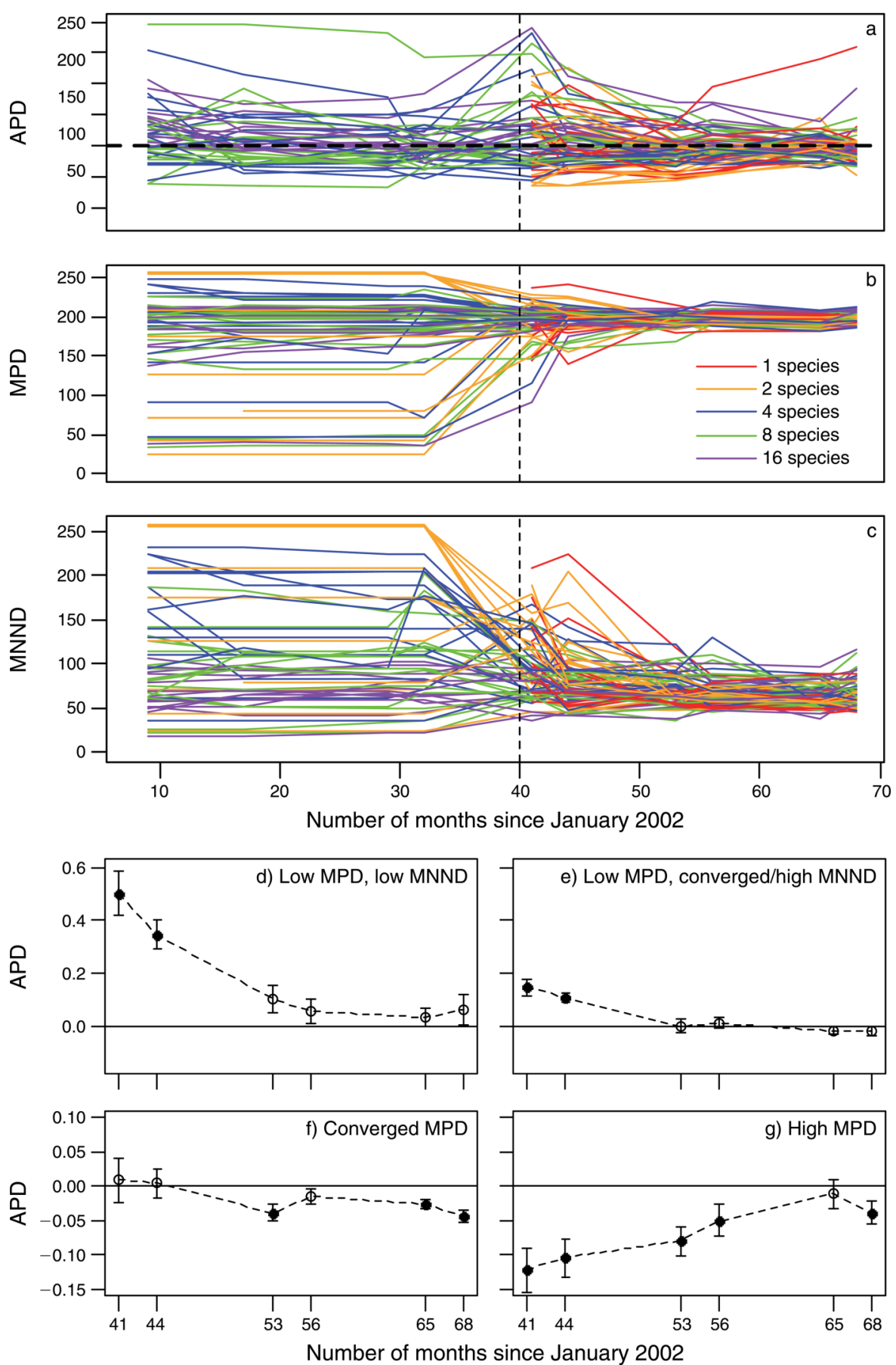

FIG. 4. (a-c) Change in phylogenetic pattern in the reassembly experiment. Colonization was allowed from April 2005 (time $=$ 40). Convergence, following colonization, in: (a) APD; (b) realized MPD (mean pairwise distance) [by summer $200795 \%$ of plots had MPD between 189 and 209 million years (Myr)]; and (c) realized MNND (mean nearest neighbor distance) [by summer 2007, 95\% of plots had MNND between 47 and $97 \mathrm{Myr}$. Each line in panels (a)-(c) represents one plot: different sown diversities are colored differently. (d-g) Mean change in APD following colonization for plots classified according to their sown MPD and MNND (in 2002) relative to $95 \%$ of the MPD and MNND values to which plots converged in summer 2007; sown values are therefore starting values and, realized phylogenetic diversity changed following colonization. Data are means \pm SE; significance is at the 5\% level. (d) Sown MPD < $189 \mathrm{Myr}$, and sown MNND < $47 \mathrm{Myr}$; (e) sown MPD < $189 \mathrm{Myr}$, and sown MNND > $47 \mathrm{Myr}$; (f) sown MPD > 189, and <209 Myr (sown MNND > $47 \mathrm{Myr}$ ); and (g) sown MPD > 209 Myr (sown MNND > 47 Myr). Open circles show plots with random phylogenetic structure (APD not significantly different from 0 ), and solid circles show those with significant phylogenetic structure (APD significantly different from 0 ). Note the $y$-axis scale in panels (d) and (e) is different from that in panels (f) and (g). 
effect $\chi^{2}=10 ; P<0.01$ ) (Fig. 4f). (4) Seventeen plots had been sown with mostly distantly related species (sown MPD higher and MNND in the range of converged values), these initially became strongly overdispersed and then APD increased (time effect, $\chi^{2}=11$; $P<0.01$ ) (Fig. 4g). This last result suggests that APD at convergence is lower than the maximum possible.

Development of overdispersion following colonization was also affected by sown species richness and functional group composition: in the first year overdispersion developed on 1-, 2- and 4-species communities, but 8 - and 16-species communities became clustered (Appendix C: Table C2). Legume presence also promoted the development of overdispersion (Appendix C: Table C2).

\section{DisCUSSION}

\section{Change in phylogenetic pattern in communities with fixed species composition}

Phylogenetic overdispersion in abundance distributions developed in experimental communities with fixed species compositions, as predicted by our first hypothesis. The relative abundances of the species in these communities differed from the ones expected based on their performance in monoculture, and by the end of the time series the dominant species in mixture were less closely related to each other than expected based on their abundance in monoculture. This would suggest that interactions between species, perhaps limiting similarity and/or facilitation, drove the increase in phylogenetic overdispersion in abundance distributions. Environmental filtering has also been shown to cause overdispersion (Cavender-Bares et al. 2006), however this would be an unlikely explanation for our results given that by the end of the time series the abundant species in polyculture communities were more distantly related to each other than were the most abundant species in monoculture. If environmental filtering drove overdispersion then the same species should become abundant in monoculture. If mixtures because all experimental communities were grown in the same environment.

Several mechanisms may have caused the development of overdispersed abundances. Phylogenetically related species may have shared ecological niches, meaning that close relatives could not coexist at high abundance over time (Maherali and Klironomos 2007). Closely related species could share similar resource (Prinzing 2001, Cahill et al. 2008) or pathogen niches (Gilbert and Webb 2007) and therefore compete more strongly with each other (Burns and Strauss 2011). More distantly related species, in contrast, may have had complementary or facilitative interactions, which is supported by the finding that fixed-composition plant communities with higher phylogenetic diversity produce more biomass (Cadotte et al. 2008). Previous work has shown an increase in species complementarity effects over time in biodiversity experiments (Cardinale et al.
2007, Marquard et al. 2009) and observational studies on succession have also shown an increase in overdispersion through time (Letcher 2009). The increase in overdispersion through time that we find, and the increasing phylogenetic diversity in mixtures vs. monocultures, indicates that limiting similarity became increasingly important as the communities assembled.

Not all communities became phylogenetically overdispersed: the development of overdispersed abundance distributions depended on the phylogenetic pattern in composition (hypothesis 2). Communities lacking close relatives did not develop overdispersed abundance distributions. These results support those of studies that have found a stronger pattern of overdispersion at small phylogenetic scales (Cavender-Bares et al. 2006, Swenson et al. 2006). Strong negative interactions may only occur between close relatives: for example, Gilbert and Webb (2007) found that the ability of the fungal pathogens of one plant species to attack another declined with phylogenetic distance between the plants, but the decline was steepest between the closest relatives. Competitive exclusion was also shown to occur more frequently and more rapidly between closely related protist species (Violle et al. 2011). In communities without close relatives present, phylogenetic distance between species may not have been a good predictor of their interactions.

Although communities without close relatives did not develop overdispersion, neither did communities composed of plants from only one family, i.e., pure grass or legume communities. These communities could in principle develop overdispersed abundance distributions because our measure of overdispersion was calculated relative to the composition of the community. There was also large variability between communities with only close relatives present, suggesting that some did develop overdispersed abundance distributions. In a microbial system functionally similar species had antagonistic interactions with each other, meaning that increasing the number of functionally similar species in a community reduced ecosystem function (Jousset et al. 2011). In our single-family communities, it is therefore conceivable that all interactions were competitive and this led to a large variability in phylogenetic pattern of abundance distributions. In communities that did develop overdispersion, positive interactions, either complementary or facilitative (Valiente-Banuet and Verdu 2007), among distantly related species may have been important. Perhaps only in communities with both close and distant relatives, where a mix of positive and negative interactions might be expected, could species abundances shift to reduce negative interactions between close relatives.

Phylogenetic diversity effects were independent of species richness: the development of overdispersed abundance distributions was not affected by species number. Greater overdispersion in species-rich communities might be expected if there is greater complementarity in these communities (Marquard et al. 2009); 
however our results show that phylogenetic diversity is more important than species richness in driving these patterns. More diverse plots did, however, develop a greater difference in phylogenetic diversity between mixture and monocultures.

Functional complementarity was probably an important mechanism underlying the development of phylogenetic overdispersion (hypothesis 3) because legume presence significantly increased overdispersion. There are two possible reasons why legumes increased overdispersion: (1) they can facilitate other species by increasing soil fertility (Temperton et al. 2007, Gubsch et al. 2011) and (2) they may be particularly sensitive to taxon-specific pathogen accumulation and/or phosphorus depletion (Roscher et al. 2011), preventing their dominance over time. However, complementarity between functional groups cannot be the only reason that phylogenetic overdispersion increases over time: after correcting for functional group presence in our statistical models, the development of overdispersion in abundance distributions still depended on sown phylogenetic diversity.

All experimental communities had phylogenetically random abundances when sown but differential recruitment and time needed for establishment (Heisse et al. 2007) led to clustered abundance distributions by the first cover survey. Traditionally, clustered patterns were thought to arise from environmental filtering on conserved species traits, and early successional or disturbed communities have been shown to be dominated by closely related species (Helmus et al. 2010) because regeneration traits are phylogenetically conserved (Burns and Strauss 2011). However by the first cover survey, the species that had become abundant in mixture were more closely related to each other than were the abundant species in monoculture. Interspecific competition may therefore have caused the increase in clustering at the beginning of the experiment (Mayfield and Levine 2010). To fully test this idea would require assessing phylogenetic signal in fitness or competitive ability measures for all species. An analysis of several commonly measured morphological and physiological traits showed that most had low phylogenetic signal (D. F. B. Flynn, E. Allan, T. Jenkins, C. Roscher, and B. Schmid, unpublished manuscript) but there were no direct measures of fitness traits. This suggests that phylogenetic distance quantifies variation in unmeasured traits. Consistent with increasing complementarity effects over time in the Jena Experiment (Marquard et al. 2009), our results suggest that only highly competitive species were able to persist initially, before niche differences became important in driving coexistence in later years.

\section{Change in phylogenetic pattern following colonization of new species}

Communities that were allowed to reassemble through colonization of new species also became overdispersed (hypothesis 4). Colonization of communities led to a convergence in phylogenetic diversity after three years, agreeing with some other recent results (Cadotte and Strauss 2011). Like Cadotte and Strauss (2011), we found that communities ended up with species-rich but distantly related clades. This suggests that community composition did not become phylogenetically overdispersed, although abundance did. This idea is further supported by the fact that overdispersion in abundance was as evident when it was calculated ignoring phylogenetic pattern in composition. Such results show the importance of looking at phylogenetic pattern in abundance distributions because they imply that closely related species can co-occur in a community but cannot both reach high abundance.

The sown phylogenetic pattern in composition did affect the change in phylogenetic pattern in abundance distributions, following colonization. Communities originally sown with only closely related species developed clustered abundance distributions immediately after colonization. This was because resident species remained dominant in the first year and these were clustered relative to the colonizers, which were still at low abundance. In some of our colonized communities, those originally lacking close relatives, overdispersion in abundance distributions briefly increased above the level to which all communities later converged. This result suggests that processes other than limiting similarity may be important in determining the identity of the species in these communities. A balance between environmental-filtering and limiting-similarity processes or between competitive ability differences and niche differences (Mayfield and Levine 2010) may therefore have led to overdispersion at convergence being lower than the maximum possible.

\section{Conclusions}

We found evidence for the development of overdispersion in abundance distributions in our experimental grassland communities, and were able to show that this was driven by interspecific competition, which suggests that limiting similarity processes become increasingly apparent as these communities reassemble. If the composition of the community was held constant, the emergence of phylogenetic overdispersion in abundance distributions depended on the presence of speciesrich but distantly related clades in the community. Interactions between both closely related and distantly related species may therefore have driven the emergence of overdispersed abundance distributions. Allowing composition to also reassemble resulted in convergence in phylogenetic diversity and in communities that were composed of several distantly related but species-rich clades and that had overdispersed abundance distributions. This suggests that limiting similarity processes determine which species dominate a community but not which species occur in a community. Crucially, as our study was carried out in experimental communities, we can rule out local evolutionary or dispersal explanations 
for these patterns and identify ecological processes as the driving force, underlining the advantages of studying these processes in experimental communities. Phylogenetic relations between species may provide a good guide to their interactions because they integrate information on hard-to-measure traits such as pathogens shared between species that would not be included in studies based on functional traits. Our results show the importance of considering phylogenetic relations between species to understand community structure, moreover, they add a new perspective to the evidence that niche complementarity is critical in driving community assembly.

\section{ACKNOWLEDGMENTS}

We thank Nicolas Salamin, Charlotte Ndiribe, and Gavin Thomas for help and advice on phylogeny reconstruction, and Dan Flynn and Tadashi Fukami for comments on an earlier version of the manuscript. We thank Elisabeth Marquard for providing some cover data and Alexandra Weigelt for her management of the Jena Experiment. We also thank the gardeners and technical staff for maintaining the site, weeding, mowing, and data collection. Many student helpers were also involved in the weeding of the experiment. This project was funded by the Deutsche Forschungsgemeinschaft (FOR 456) with additional contributions from the Swiss National Science Foundation (grant 31003A-107531 to B. Schmid).

\section{Literature Cited}

APG [Angiosperm Phylogeny Group]. 2009. An update of the Angiosperm Phylogeny Group classification for the orders and families of flowering plants: APG III. Botanical Journal of the Linnean Society 161:105-121.

Bates, D., M. Maechler, and B. M. Bolker. 2011. Lme4: linear mixed-effects models using S4 classes. Version 0.999375-42. http://lme4.r-forge.r-project.org/

Benson, D. A., M. S. Boguski, D. J. Lipman, J. Ostell, B. F. F. Ouellette, B. A. Rapp, and D. L. Wheeler. 1999. GenBank Nucleic Acids Research 27:12-17.

Burns, J. H., and S. Y. Strauss. 2011. More closely related species are more ecologically similar in an experimental test. Proceedings of the National Academy of Sciences USA 108:5302-5307.

Cadotte, M. W., B. J. Cardinale, and T. H. Oakley. 2008. Evolutionary history and the effect of biodiversity on plant productivity. Proceedings of the National Academy of Sciences USA 105:17012-17017.

Cadotte, M. W., and S. Y. Strauss. 2011. Phylogenetic patterns of colonization and extinction in experimentally assembled plant communities. PLoS One 6:e19363.

Cahill, J. F., S. W. Kembel, E. G. Lamb, and P. A. Keddy. 2008. Does phylogenetic relatedness influence the strength of competition among vascular plants? Perspectives in Plant Ecology, Evolution and Systematics 10:41-50.

Cardinale, B. J., J. P. Wright, M. W. Cadotte, I. T. Carroll, A. Hector, D. S. Srivastava, M. Loreau, and J. J. Weis. 2007. Impacts of plant diversity on biomass production increase through time because of species complementarity. Proceedings of the National Academy of Sciences USA 104:1812318128.

Cavender-Bares, J., A. Keen, and B. Miles. 2006. Phylogenetic structure of Floridian plant communities depends on taxonomic and spatial scale. Ecology 87:109-122.

Cavender-Bares, J., K. H. Kozak, P. V. A. Fine, and S. W. Kembel. 2009. The merging of community ecology and phylogenetic biology. Ecology Letters 12:693-715.

Cingolani, A. M., M. Cabido, D. E. Gurvich, D. Renison, and S. Diaz. 2007. Filtering processes in the assembly of plant communities: Are species presence and abundance driven by the same traits? Journal of Vegetation Science 18:911-920.

Cornwell, W. K., and D. D. Ackerly. 2010. A link between plant traits and abundance: evidence from coastal California woody plants. Journal of Ecology 98:814-821.

Crawley, M. J. 2007. The R book. John Wiley and Sons, Chichester, UK.

Darwin, C. 1859. The origin of species by means of natural selection. Murray, London, UK.

Drummond, A. J., S. Y. W. Ho, M. J. Phillips, and A. Rambaut. 2006. Relaxed phylogenetics and dating with confidence. PLoS Biology 4(5):e88.

Drummond, A. J., and A. Rambaut. 2007. BEAST: Bayesian evolutionary analysis by sampling trees. BMC Evolutionary Biology 7:214

Edgar, R. C. 2004. MUSCLE: multiple sequence alignment with high accuracy and high throughput. Nucleic Acids Research 32:1792-1797.

Fukami, T., T. M. Bezemer, S. R. Mortimer, and W. H. Putten. 2005. Species divergence and trait convergence in experimental plant community assembly. Ecology Letters 8:1283-1290.

Gilbert, G. S., and C. O. Webb. 2007. Phylogenetic signal in plant pathogen-host range. Proceedings of the National Academy of Sciences USA 104:4979-4983.

Gubsch, M., C. Roscher, G. Gleixner, M. Habekost, A. Lipowsky, B. Schmid, E.-D. Schulze, S. Steinbeiss, and N. Buchmann. 2011. Foliar and soil $\delta 15 \mathrm{~N}$ values reveal increased nitrogen partitioning among species in diverse grassland communities. Plant, Cell and Environment 34:895908.

Hardy, O. J. 2008. Testing the spatial phylogenetic structure of local communities: statistical performances of different null models and test statistics on a locally neutral community. Journal of Ecology 96:914-926.

Hardy, O. J., and B. Senterre. 2007. Characterizing the phylogenetic structure of communities by an additive partitioning of phylogenetic diversity. Journal of Ecology 95:493-506.

Heisse, K., C. Roscher, J. Schumacher, and E.-D. Schulze. 2007. Establishment of grassland species in monocultures: different strategies lead to success. Oecologia 152:435-447.

Helmus, M. R., W. Keller, M. J. Paterson, N. D. Yan, C. H. Cannon, and J. A. Rusak. 2010. Communities contain closely related species during ecosystem disturbance. Ecology Letters $13: 162-174$.

Jousset, A., B. Schmid, S. Scheu, and N. Eisenhauer. 2011. Genotypic richness and dissimilarity opposingly affect ecosystem functioning. Ecology Letters 14:537-545.

Kembel, S. W. 2009. Disentangling niche and neutral influences on community assembly: assessing the performance of community phylogenetic structure tests. Ecology Letters 12:949-960.

Kembel, S. W., P. D. Cowan, M. R. Helmus, W. K. Cornwell, H. Morlon, D. D. Ackerly, S. P. Blomberg, and C. O. Webb. 2010. Picante: $\mathrm{R}$ tools for integrating phylogenies and ecology. Bioinformatics 26:1463-1464.

Kraft, N. J. B., and D. D. Ackerly. 2010. Functional trait and phylogenetic tests of community assembly across spatial scales in an Amazonian forest. Ecological Monographs 80:401-422.

Letcher, S. G. 2009. Phylogenetic structure of angiosperm communities during tropical forest succession. Proceedings of the Royal Sociey B 277:97-104.

Loreau, M., and A. Hector. 2001. Partitioning selection and complementarity in biodiversity experiments. Nature 412:7276.

Maherali, H., and J. N. Klironomos. 2007. Influence of phylogeny on fungal community assembly and ecosystem functioning. Science 316:1746-1748.

Marquard, E., A. Weigelt, V. M. Temperton, C. Roscher, J. Schumacher, N. Buchmann, M. Fischer, W. W. Weisser, and 
B. Schmid. 2009. Plant species richness and functional composition drive overyielding in a six-year grassland experiment. Ecology 12:3290-3302.

Mayfield, M. M., and J. M. Levine. 2010. Opposing effects of competitive exclusion on the phylogenetic structure of communities. Ecology Letters 13:1085-1093.

Pacala, S. W., and D. Tilman. 1994. Limiting similarity in mechanistic and spatial models of plant competition in heterogeneous environments. American Naturalist 143:222257.

Petermann, J. S., A. J. F. Fergus, C. Roscher, L. A. Turnbull, A. Weigelt, and B. Schmid. 2010. Biology, chance, or history? The predictable reassembly of temperate grassland communities. Ecology 91:408-421.

Pfisterer, A. B., J. Joshi, B. Schmid, and M. Fischer. 2004. Rapid decay of diversity-productivity relationships after invasion of experimental plant communities. Basic and Applied Ecology 5:5-14.

Posada, D., and K. A. Crandall. 1998. MODELTEST: testing the model of DNA substitution. Bioinformatics 14:817-818.

Prinzing, A. 2001. The niche of higher plants: evidence for phylogenetic conservatism. Proceedings of the Royal Society B 268:2383-2389.

R Development Core Team. 2010. R: a language and environment for statistical computing. R Foundation for Statistical Computing, Vienna, Austria.

Roscher, C., B. Schmid, and E.-D. Schulze. 2009a. Nonrandom recruitment of invader species in experimental grasslands. Oikos 118:1524-1540.

Roscher, C., J. Schumacher, J. Baade, W. Wilcke, G. Gleixner, W. W. Weisser, B. Schmid, and E. D. Schulze. 2004. The role of biodiversity for element cycling and trophic interactions: an experimental approach in a grassland community. Basic and Applied Ecology 5:107-121.

Roscher, C., V. M. Temperton, N. Buchmann, and E.-D. Schulze. 2009b. Community assembly and biomass production in regularly and never weeded experimental grasslands. Acta Oecologica-International Journal of Ecology 35:206217.

Roscher, C., S. Thein, A. Weigelt, V. Temperton, N. Buchmann, and E.-D. Schulze. 2011. $\mathrm{N}_{2}$ fixation and performance of 12 legume species in a 6-year grassland biodiversity experiment. Plant and Soil 341(1-2):333-348.

Stubbs, W. J., and J. B. Wilson. 2004. Evidence for limiting similarity in a sand dune community. Journal of Ecology 92:557-567.

Swenson, N. G., B. J. Enquist, J. Pither, J. Thompson, and J. K. Zimmerman. 2006. The problem and promise of scale dependency in community phylogenetics. Ecology 87:24182424.

Temperton, V. M., P. N. Mwangi, M. Scherer-Lorenzen, B. Schmid, and N. Buchmann. 2007. Positive interactions between nitrogen-fixing legumes and four different neighbouring species in a biodiversity experiment. Oecologia 151:190-205.

Thompson, K., O. L. Petchey, A. P. Askew, N. P. Dunnett, A. P. Beckerman, and A. J. Willis. 2010. Little evidence for limiting similarity in a long-term study of a roadside plant community. Journal of Ecology 98:480-487.

Valiente-Banuet, A., and M. Verdu. 2007. Facilitation can increase the phylogenetic diversity of plant communities. Ecology Letters 10:1029-1036.

Vamosi, S. M., S. B. Heard, J. C. Vamosi, and C. O. Webb. 2009. Emerging patterns in the comparative analysis of phylogenetic community structure. Molecular Ecology 18:572-592.

Violle, C., D. R. Nemergut, Z. Pu, and L. Jiang. 2011. Phylogenetic limiting similarity and competitive exclusion. Ecology Letters 14(8):782-787.

Webb, C. O., D. D. Ackerly, M. A. McPeek, and M. J. Donoghue. 2002. Phylogenies and community ecology. Annual Review of Ecology and Systematics 33:475-505.

Webb, C. O., G. S. Gilbert, and M. J. Donoghue. 2006. Phylodiversity-dependent seedling mortality, size structure, and disease in a Bornean rain forest. Ecology 87:123-131.

Weigelt, A., E. Marquard, V. M. Temperton, C. Roscher, C. Scherber, P. N. Mwangi, S. Felten, N. Buchmann, B. Schmid, E.-D. Schulze, and W. W. Weisser. 2010. The Jena Experiment: six years of data from a grassland biodiversity experiment. Ecology 91:930-931.

\section{Supplemental Material}

\section{Appendix A}

A table presenting information about the angiosperm fossils used in the molecular phylogenetic tree calibration (Ecological Archives E094-038-A1).

\section{Appendix B}

Details on the calculation of APD and $D_{\text {diff }}^{\mathrm{B}}$ and change in $D_{\text {diff }}^{\mathrm{B}}$ over time in the fixed composition experiment (Ecological Archives E094-038-A2).

\section{Appendix C}

One table and two figures presenting the correlation between sown species richness and sown phylogenetic diversity plus the minimal adequate models for the analysis of the fixed composition and reassembly experiments (Ecological Archives E094-038-A3).

\section{Appendix D}

The effect of calculating APD in the reassembly experiment ignoring phylogenetic pattern in colonization (Ecological Archives E094-038-A4). 